\title{
STABILITY RESULTS FOR A DIFFUSION EQUATION WITH FUNCTIONAL DRIFT APPROXIMATING A CHEMOTAXIS MODEL ${ }^{1}$
}

\author{
JAMES M. GREENBERG AND WOLFGANG ALT
}

\begin{abstract}
A hyperbolic-parabolic "chemotaxis" system modelling aggregation of motile cells by production of a diffusible chemoattractant, is approximated by a scalar diffusion equation for the cell density, where the drift term is an explicit functional of the current density profile. We prove the unique existence and, using the Hopf-Cole transformation, the local stability of an equilibrium, i.e. a steady aggregation state. We also discuss the limiting hyperbolic case of vanishing random motility with the formation of shocks describing cell clumps.
\end{abstract}

1. Introduction and development of the model. In this note we shall develop and explore the behavior of a simple two-speed, one-dimensional model which describes the motion of chemosensitive cells; that is cells whose motions are influenced by a chemical gradient $\partial \rho / \partial x$, where $\rho=\rho(x, t)$ denotes the concentration of a diffusible substance produced by each cell. A well-known example is the morphogenetic aggregation of slime mold cells [5], which previously has been modelled by Keller and Segel [6] using a coupled system of two equations for $\rho$ and the cell density $u=u(x, t)$, the so-called "chemotaxis system". For a recent mathematical analysis see $[4, \mathbf{1 2}]$. In most cases as in slime molds, however, parameter estimations reveal that the diffusion coefficient of the chemoattractant $\left(D_{\rho} \sim 10^{-7} \mathrm{~cm}^{2} / \mathrm{sec}\right)$ is about ten times larger than the motility of the cells $\left(D_{u} \sim 10^{-8} \mathrm{~cm}^{2} / \mathrm{sec}\right)$ (compare for example [3]). If also the production rate of the chemoattractant is relatively large, then the quasi-stationary gradient of the rapidly diffusing substance approximately determines the chemotactic drift of the cells. This finally leads to a scalar diffusion equation for the cell density $u$ of type

$$
\frac{\partial u}{\partial t}+\frac{\partial}{\partial x}\left\{u V-K^{2} \frac{\partial u}{\partial x}\right\}=0
$$

where the chemotactic drift velocity $V(x, t)$ is a functional of the momentary cell density $u(\cdot, t)$ at time $t$. For a more general treatment of these kind of equations see $[\mathbf{1}, \mathbf{2}, \mathbf{8}]$.

In this first section we want to give a brief derivation of (1.1) from a basic stochastic model, and then proceed with its analysis. After rescaling space and

Received by the editors July 31,1985 .

1980 Mathematics Subject Classification. Primary 35B35, 35L65.

Key words and phrases. Diffusion equation with functional, chemotaxis model, Hopf-Cole transformation, shock waves.

${ }^{1}$ This work has been supported by the Deutsche Forschungsgemeinschaft and the U.S. National Science Foundation under grant MCS 8219806. 
time the one-dimensional motion of cells with speed $\pm c$ can be described by a probability density function $f:\{-c, c\} \times[-1,1] \times[0, \infty) \rightarrow[0, \infty)$. The values

$$
f^{-} \stackrel{\text { def }}{=} f(-c, x, t) \geq 0 \quad \text { and } \quad f^{+} \stackrel{\text { def }}{=} f(c, x, t) \geq 0
$$

represent the probability density of finding a cell at $x$ at time $t$ with speeds $-c$ and $+c$ respectively (compare $[\mathbf{1 0}]$ ). The evolution equations for $f^{-}$and $f^{+}$are

$$
\frac{\partial f^{-}}{\partial t}-c \frac{\partial f^{-}}{\partial x}=-\frac{1}{2}\left(\frac{1}{\mu}+\chi \frac{\partial \rho}{\partial x}\right) f^{-}+\frac{1}{2}\left(\frac{1}{\mu}-\chi \frac{\partial \rho}{\partial x}\right) f^{+}
$$

and

$$
\frac{\partial f^{+}}{\partial t}+c \frac{\partial f^{+}}{\partial x}=-\frac{1}{2}\left(\frac{1}{\mu}-\chi \frac{\partial \rho}{\partial x}\right) f^{+}+\frac{1}{2}\left(\frac{1}{\mu}+\chi \frac{\partial \rho}{\partial x}\right) f^{-},
$$

where $\chi$ and $\mu$ are positive constants. The terms $1 / \mu \pm \chi(\partial \rho / \partial x)$ in (1.2) and (1.3) represent the fact that, without a gradient of $\rho$, a single cell moving with one speed $( \pm c)$ randomly switches to the other in a time interval of length $d t$ with probability $1 / 2 \mu d t$. In presence of a chemical gradient this probability is increased (or decreased) for cells moving down (or up) the gradient. For simplicity we assume a linear dependence on $\partial \rho / \partial x$.

Introducing the two moments

$$
\begin{aligned}
u & =f^{+}+f^{-} \text {the total cell density, } \\
w & =c\left(f^{+}-f^{-}\right) \text {the mean cell flux, }
\end{aligned}
$$

it is easily checked that (1.2)-(1.3) is equivalent to the hyperbolic system (see also [10]):

$$
\begin{aligned}
& \frac{\partial u}{\partial t}+\frac{\partial w}{\partial x}=0, \\
& \frac{\partial w}{\partial t}+c^{2} \frac{\partial u}{\partial z}=-\frac{w}{\mu}+c \chi u \frac{\partial \rho}{\partial x} .
\end{aligned}
$$

The model system is closed by a parabolic diffusion equation for $\rho$ with a production rate proportional to $u$;

$$
\varepsilon \frac{\partial \rho}{\partial t}=d \frac{\partial^{2} \rho}{\partial x^{2}}+u
$$

where $\varepsilon$ and $d$ are positive constants.

Our primary interest is in solutions of (1.6)-(1.8) in a finite tube: $-1<x<1$ for times $t>0$, given initial data for $u, w$, and $\rho$ at time $t=0$. (Due to the preliminary rescaling of space we can choose the spatial interval $[-1,1]$ without restriction.) This requires that boundary conditions be supplied at the ends $x= \pm 1$. We shall assume zero boundary conditions for the cell flux $w$;

$$
w( \pm 1, t)=0
$$

and that $\rho$ satisfies the symmetric Robin conditions;

$$
\frac{\partial \rho}{\partial x}(-1, t)=\Lambda \rho(-1, t) \quad \text { and } \quad \frac{\partial \rho}{\partial x}(1, t)=-\Lambda \rho(1, t)
$$

where $\Lambda>0$ measures the dilution of the chemoattractant into the exterior. 
We first observe that if $(u, w, \rho)$ is a solution of $(1.6)-(1.10)$, then the cumulative distribution function

$$
U(x, t) \stackrel{\text { def }}{=} \int_{-1}^{x} u(\xi, t) d \xi
$$

satisfies

$$
\frac{\partial U}{\partial t}+w=0, \quad-1<x<1 \text { and } t>0 .
$$

Moreover, $w(1, t)=0$ implies that, besides $U(-1, t) \equiv 0$,

$$
U(1, t) \equiv 2 \bar{u}, \quad t>0 .
$$

Here

$$
\bar{u}=\frac{1}{2} \cdot \int_{-1}^{1} u(x, t) d x
$$

is the mean value of $u(\cdot, t)$ which is independent of $t$.

The assumption mentioned at the beginning, that diffusion and production of the chemoattractant are large (relative to the macroscopic scales), means for equation (1.8) that $\varepsilon$ is vanishingly small, more precisely we will suppose

$$
0<\varepsilon \ll d \lesssim 1 \text {. }
$$

This first assumption allows us, in the asymptotic limit $\varepsilon \rightarrow 0$, to replace (1.8) by the quasi-stationary approximation for the gradient;

$$
d \frac{\partial \rho}{\partial x}=b(t)-U(x, t), \quad-1 \leq x \leq 1 \text { and } t>0,
$$

where

$$
b(t)=\frac{2 \bar{u}+\Lambda \int_{-1}^{1} U(\xi, t) d \xi}{2(1+\Lambda)}, \quad t>0
$$

contains the double integral functional of $u(\cdot, t)$.

Using (1.16) we can replace the gradient $\partial \rho / \partial x$ in (1.6)-(1.7) and obtain an approximating hyperbolic system with a functional term;

$$
\begin{gathered}
\frac{\partial u}{\partial t}+\frac{\partial w}{\partial x}=0 \\
\mu \frac{\partial w}{\partial t}+\mu c^{2} \frac{\partial u}{\partial x}=-w+\frac{\mu c \chi}{d} u(b(t)-U) .
\end{gathered}
$$

In particular, we want to analyze situations where the net chemotactic drift of the cells, described by the quotient $\mu c \chi / d$ in equation (1.18), is of order 1 . By an appropriate choice of the preliminary time rescaling we might, without restriction, suppose that

$$
\mu c \chi=d .
$$

Then the motility (diffusion coefficient) for the cells is given by

$$
K^{2}=\mu c^{2}=c d / \chi .
$$


Since an analysis of the full hyperbolic system (1.18), (1.19) with boundary conditions (1.9) is not yet amenable, we additionally suppose that the random switching frequency $1 / \mu$ is relatively large, i.e.

$$
0<\mu \ll 1 .
$$

Then we obtain from (1.19) the following diffusive approximation for the flux $w$;

$$
w=u(b(t)-U)-K^{2} \frac{\partial u}{\partial x} .
$$

Replacing $w$ in (1.18) or (1.12) respectively, we finally arrive at the diffusion equation (1.1) for the cell density $u$

$$
\frac{\partial u}{\partial t}+\frac{\partial}{\partial x}\left[u(b(t)-U)-K^{2} \frac{\partial u}{\partial x}\right]=0
$$

or an analogous diffusion equation for the cumulative distribution function $U$ in (1.11);

$$
\frac{\partial U}{\partial t}+\frac{\partial}{\partial x}\left[b(t) U-\frac{1}{2} U^{2}-K^{2} \frac{\partial U}{\partial x}\right]=0 .
$$

Equation (1.24) is supplied by the Neumann flux condition $w=0$ with $w$ in (1.23). Equivalently, this gives the following Dirichlet condition for the parabolic equation (1.25), see also (1.13);

$$
U(-1, t) \equiv 0 \quad \text { and } \quad U(+1, t) \equiv 2 \bar{u} .
$$

Notice that (1.25) still contains the functional term

$$
b(t) \stackrel{\text { def }}{=} \frac{2 \bar{u}+\Lambda \int_{-1}^{1} U(\xi, t) d \xi}{2(1+\Lambda)} .
$$

If we formally (for $\mu=\varepsilon=0$ ) proceed to the limit $\Lambda=0$ we observe that $b(t) \equiv \bar{u}$ is constant and we are back to a classical nonlinear diffusion problem which, in the case of the infinite real line and with density dependent degenerate diffusion coefficient $K^{2}$, has been analyzed by Mimura and Nagai [8, 9]. Remark, however, that $\rho \sim b / \Lambda$ for $\varepsilon=0$ and $\Lambda \rightarrow 0$, or $\int_{-1}^{1} \rho(t, x) d x \sim \bar{u} / 2 \varepsilon \cdot t$ for $\varepsilon>0$ and $\Lambda=0$ in (1.10).

The remainder of this paper involves a systematic study of system (1.25)-(1.27) for $-1<x<1$ and $t>0$, with initial data

$$
U(x, 0)=\hat{U}(x), \quad-1 \leq x \leq 1,
$$

where $\hat{U}(\cdot)$ is any piecewise smooth function satisfying the compatibility conditions

$$
\hat{U}(-1)=0, \quad \hat{U}(1)=2 \bar{u}, \quad \text { and } \quad \frac{d \hat{U}}{d x}(x) \geq 0, \quad-1 \leq x \leq 1 .
$$

Our principal result is that for all $\Lambda \geq 0$ and positive $K^{2}$ in (1.21) a unique equilibrium profile is obtained (Proposition 1 ) which is locally stable (Theorem 2 ). For small values of $K^{2}$ this equilibrium profile, $U_{\text {eq }}$, is approximately the step function

$$
U_{\mathrm{eq}}(x)=\left\{\begin{array}{l}
0, \\
2 \bar{u}, \quad 0<x<1
\end{array}\right.
$$


and the density $u_{\mathrm{eq}}=d U_{\mathrm{eq}} / d x$ is approximately the dirac measure at the origin

$$
u_{\mathrm{eq}}(x)=2 \bar{u} \delta(x)
$$

For $K^{2} \gg 1$, we have

$$
U_{\text {eq }}(x) \doteq \bar{u}(x+1) \quad \text { and } \quad u_{\text {eq }}=\frac{d U_{\text {eq }}}{d x} \doteq \bar{u} .
$$

Our numerical computations suggest that the unique equilibrium is also globally attractive, see $\S 4$.

These results imply that when the chemotactic effects dominate the chemical diffusion $(\chi / c \gg d)$ the cells tend to aggregate at the center of the tube whereas when chemical diffusion dominates there is a tendency of the cells to smear to a uniform distribution.

In $\S 2$ we shall investigate $(1.25)-(1.28)$ when $K^{2}=0$. In this case (1.25) reduces to a scalar quasilinear hyperbolic conservation law and solutions with shocks must be considered. The theory of such equations is well understood; for details see e.g. [7]. The interesting feature about this case is that after a finite time the solution $U$ is a step function with a single discontinuity; i.e.

$$
U(x, t)= \begin{cases}0, & -1<x<s(t) \\ 2 \bar{u}, & s(t)<x<1\end{cases}
$$

and $x=s(t)$ is a shock wave satisfying

$$
\frac{d s}{d t}=-\frac{\Lambda \bar{u} s}{1+\Lambda} \text {. }
$$

In $\S 3$ we shall analyze the system when $K^{2}>0$. Here, we shall use the Hopf-Cole transformation to simplify the problem. Spectral methods will then be applied to the transformed systems to obtain the long-term asymptotics.

2. The case $K^{2}=0$. In this section we shall study the system:

$$
\begin{gathered}
\frac{\partial U}{\partial t}+\frac{\partial}{\partial x}\left(b(t) U-\frac{U^{2}}{2}\right)=0, \quad-1<x<1 \text { and } t>0 \\
b(t)=\frac{2 \bar{u}+\Lambda \int_{-1}^{1} U(\xi, t) d \xi}{2(1+\Lambda)} \\
U(-1, t)=0 \text { and } U(1, t)=2 \bar{u},
\end{gathered}
$$

and

$$
U\left(x, 0^{+}\right)=\hat{U}(x), \quad-1<x<1,
$$

where $\hat{U}(\cdot)$ is any piecewise smooth function satisfying the compatibility conditions

$$
\hat{U}(-1)=0, \quad \hat{U}(1)=2 \bar{u}, \quad \frac{d \hat{U}}{d x}(x) \geq 0, \quad-1<x<1,
$$

and $\Lambda$ and $\bar{u}$ are positive constants.

The equation (2.1) is hyperbolic, and in regions of $-1<x<1$ and $t>0$ where $U$ is smooth, it is equivalent to the characteristic system

$$
\frac{d U}{d t}(x(t), t)=0 \text { on the curves } \frac{d x}{d t}=b(t)=U(x(t), t) .
$$


The last equation, together with the definition of $b(\cdot)$ and the boundary conditions (2.3), implies that characteristics emanating from the boundaries $x= \pm 1$ point into the interval $-1<x<1$ as $t$ increases, and this observation confirms that boundary conditions at both ends of the interval are required to make the problem properly posed.

The presence of the quadratic nonlinearity in (2.1) forces us to consider solutions which are piecewise smooth and exhibit simple jump discontinuities (in $x$ and $t$ ) across piecewise smooth curves $x=s(t)$. Such curves are called shock waves and in order that they be consistent with the conservation structure of (2.1) they must satisfy the Rankine-Hugoniot condition,

$$
\frac{d s}{d t}=b(t)-\frac{U_{+}(s(t), t)+U_{-}(s(t), t)}{2}
$$

where

$$
U_{ \pm}(s(t), t)=\lim _{\substack{\varepsilon \rightarrow 0^{+} \\ \varepsilon>0}} U(s(t) \pm \varepsilon, t) .
$$

In general, not all shock waves are admissible. However, since we look for functions $U$ being monotone in $x$, we can consider only those shocks which satisfy

$$
U_{-}(s(t), t)<U_{+}(s(t), t) .
$$

This last condition guarantees that the sound speeds $V_{-}(s(t), t)=b(t)-U_{-}(s(t), t)$ and $V_{+}(s(t), t)=b(t)-U_{+}(s(t), t)$ to the left and right of the shock satisfy

$$
V_{+}(s(t), t)<\frac{d s}{d t}<V_{-}(s(t), t) .
$$

(2.9) also guarantees that any shock solution is obtainable by taking limits as $K^{2}$ tends to zero of solutions of (1.25); clearly a desired property for us. Compare e.g. [11] for conservation laws without functional terms.

We shall now show how to solve the system (2.1)-(2.4) when the initial data $U$ is a step function; that is when

$$
U(x)= \begin{cases}U_{1}, & -1<x<s_{1}^{0} \\ U_{k}, & s_{k-1}^{0}<x<s_{k}^{0}, 2 \leq k \leq n-1, \\ U_{n}, & s_{n-1}^{0}<x<1,\end{cases}
$$

and

$$
0 \leq U_{1}<U_{2}<\cdots<U_{n} \leq 2 \bar{u} .
$$

In this case shocks will emanate from the points $x=-1, x=s_{k}^{0}, k=1,2, \ldots, n-1$, and $x=1$ and will be denoted by $x=l(t), x=s_{k}(t), k=1,2, \ldots, n-1$, and $x=r(t)$ respectively. They satisfy

$$
\begin{gathered}
\frac{d l}{d t}=b(t)-\frac{U_{1}}{2} \quad \text { and } \quad l(0)=-1 . \\
\frac{d s_{k}}{d t}=b(t)-\frac{U_{k}+U_{k+1}}{2} \text { and } s_{k}(0)=s_{k}^{0}, \quad 1 \leq k \leq n-1,
\end{gathered}
$$

and

$$
\frac{d r}{d t}=b(t)-\frac{2 \bar{u}+U_{n}}{2}, \quad r(0)=1,
$$


where

$$
b(t)=\frac{2 \bar{u}+\Lambda\left(U_{1}\left(s_{1}-l\right)+\sum_{k=2}^{n-1} U_{k}\left(s_{k}-s_{k-1}\right)+U_{n}\left(r-s_{n-1}\right)+2 \bar{u}(1-r)\right)}{2(1+\Lambda)}
$$

The solution, $U$, is given by

$$
U(x, t)= \begin{cases}0, & -1<x<l(t), \\ U_{1}, & l(t)<x<s_{1}(t), \\ U_{k}, & s_{k-1}(t)<x<s_{k}(t), 2 \leq k \leq n-1, \\ U_{n}, & s_{n-1}(t)<x<r(t), \\ 2 \bar{u}, & r(t)<x<1,\end{cases}
$$

and it is valid so long as none of the shocks have collided. When a collision occurs we simply relabel the shocks and states and continue the solution. In this process we always reserve the labels $x=l(t)$ and $x=r(t)$ for the distinguished shocks satisfying $U \equiv 0$ for $-1<x<l(t)$ and $U \equiv 2 \bar{u}$ for $r(t)<x<1$. It is clear that when collisions occur, the number of states and shocks decrease by at least one. More importantly, we find that for times $t \geq 2 / \bar{u}$ the only states remaining in the solution are 0 and $2 \bar{u}$ and that the curves $x=l(t)$ and $x=r(t)$ have merged. To see that this last statement is true we simply subtract (2.13) from (2.15) to obtain

$$
\frac{d}{d t}(r-l)=-\bar{u}-\frac{U_{n}-U_{1}}{2} .
$$

The inequalities (2.12) then yield

$$
\frac{d}{d t}(r-l) \leq-\bar{u} \quad \text { and } \quad 0 \leq r-l \leq 2-\bar{u} t
$$

which implies the result claimed.

Finally, for times $t \geq 2 / \bar{u}$ the solution is given by

$$
U(x, t)= \begin{cases}0, & 0<x<s(t), \\ 2 \bar{u}, & s(t)<x<1,\end{cases}
$$

where $s$ satisfies, by calculation of $b$ in $(2.16)$ :

$$
\frac{d s}{d t}=-\frac{\Lambda \bar{u} s}{1+\Lambda} \text {. }
$$

The solution to (2.1)-(2.4) for nonstep function data $\hat{U}$ satisfying (2.5) is obtained by first approximating $\hat{U}$ by step data, then solving the resulting problem with the approximating step data, and finally by extracting a convergent subsequence of approximate solutions which converge strongly in $L_{1}$ of any compact subset of $\{(x, t) \mid-1<x<1$ and $t>0\}$ to a weak solution of (2.1)-(2.4). That such a program is possible follows from two facts: (1) the approximating step data converge strongly in $L_{1}(-1,1)$ to the given data $\hat{U}$, and (2) the approximating solutions are monotone increasing in $x$ and satisfy the boundary conditions (2.3).

Remark that (with $\Lambda>0$ ) the final shock curves with the whole cell mass concentrated on them

$$
u(t, x)=2 \bar{u} \delta_{s(t)}(x)
$$


converge to the center of the interval as

$$
s(t) \sim e^{-\Lambda \bar{u} t_{\prime}^{\prime}(1+\Lambda)} \rightarrow 0 \quad \text { as } t \rightarrow \infty .
$$

This convergence, however, is not uniform in $\Lambda>0$ since for the limiting cases $\Lambda=0,(2.1)$ reduces to the well-known Hopf equation whose final shock lines are constant in time, their position depending on the initial data. For small $K^{2}>0$ compare the numerical simulation in Figure 2b, which approximates the hyperbolic case $K^{2}=0$.

3. The case $K^{2}>0$. We now turn to the problem described in (1.25)-(1.28). Here it is convenient to introduce a new potential $W$ defined by

$$
W \stackrel{\text { def }}{=} U-\bar{u} \text {. }
$$

Then, solving (1.25)-(1.28) is equivalent to solving

$$
\frac{\partial W}{\partial t}+\frac{\partial}{\partial x}\left(B(t) W-\frac{W^{2}}{2}\right)-K^{2} \frac{\partial^{2} W}{\partial x^{2}}=0, \quad-1<x<1, t>0
$$

where $B$ is given by

$$
B(t)=\frac{\Lambda}{2(1+\Lambda)} \int_{-1}^{1} W(\xi, t) d \xi
$$

and $W$ satisfies

$$
W(-1, t)=-\bar{u} \quad \text { and } \quad W(1, t)=\bar{u}
$$

and

$$
W\left(x, 0^{+}\right)=\hat{U}(x)-\bar{u} \stackrel{\text { def }}{=} \hat{W}(x), \quad-1<x<1 .
$$

First let us find the equilibria of this system. Any time independent solution $W=W(x)$ with $W^{\prime} \geq 0$ can be written as

$$
W(x)=Z(x)+B
$$

where $Z$ satisfies the Riccati equation

$$
Z^{\prime}=-\frac{Z^{2}-C^{2}}{2 K^{2}} \quad \text { for some constant } C \geq 0
$$

with boundary conditions

$$
Z( \pm 1)= \pm \bar{u}-B
$$

and

$$
B=\frac{\Lambda}{2} \int_{-1}^{1} Z
$$

Integration of (3.7) yields

$$
Z(x)=C \frac{1-A e^{-C x / K^{2}}}{1+A e^{-C x / K^{2}}}
$$

with some constant $A>0$. The free integration constants $A$ and $C$ are determined by (3.8) and (3.9) via the following conditions for

$$
a^{ \pm} \stackrel{\text { def }}{=} A e^{ \pm C / K^{2}}
$$


(a)

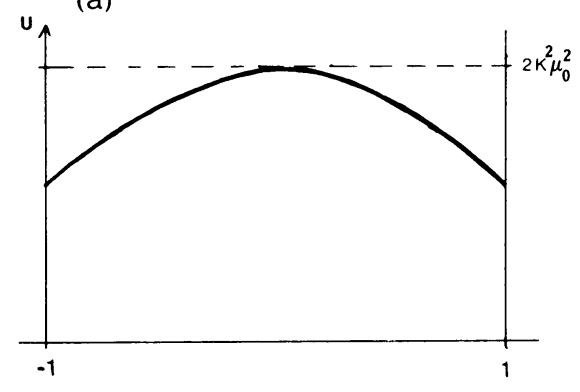

(b)

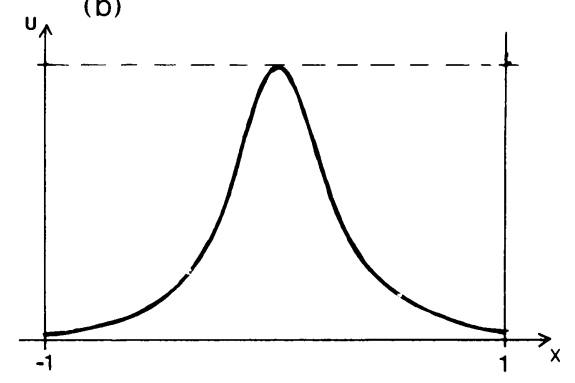

FIGURE 1. The unique solution $u=u_{\text {eq }}(x)$ given by (3.17) for different parameter choices with equal maximal value of $u_{\text {eq }}$. A broad distribution (a) is achieved by a large diffusion constant $K^{2}$ resulting in a small $\mu_{0}$, whereas for small $K^{2}$, i.e. large $\mu_{0}$, the distribution is clustered around the origin (b). Thus, with fixed $K^{2}$ shape (a) arises for small mean densities $\bar{u}$, whereas (b) is obtained for large $\bar{u}$.

namely

$$
\Lambda\left\{1-2 \ln \frac{1+a^{-}}{1+a^{+}} / \ln \frac{a^{-}}{a^{+}}\right\}=\frac{a^{+} a^{-}-1}{\left(1+a^{+}\right)\left(1+a^{-}\right)}
$$

and

$$
\ln \frac{a^{+}}{a^{-}} \frac{a^{+}-a^{-}}{\left(1+a^{+}\right)\left(1+a^{-}\right)}=\frac{2 \bar{u}}{K^{2}} .
$$

It turns out that the zero curve $\left\{\left(a^{+}, a^{-}\right): a^{+} a^{-}=1\right\}$ of both the right and the left sides of equation (3.12) represents all solutions, since the two functions have opposite sign on each side of the zero curve. Now $a^{-}=1 / a^{+}$corresponds via (3.11) to $A=1$ in (3.10) and $B=0$ in (3.9). Then condition (3.13) determines the second constant to be

$$
C=2 K^{2} \mu_{0}
$$

where

$$
0<\mu_{0} \text { is such that } \mu_{0} \tanh \mu_{0}=\bar{u} / 2 K^{2} \text {. }
$$

Thus, we have the following

PROPOSITION 1 (UNIQUE EQUILIBRIUM). With $\Lambda \geq 0$ and $K^{2}>0$ system (3.2)-(3.5) has, for given mean value $\bar{u}$, a unique equilibrium solution

$$
W_{\text {eq }}(x)=2 K^{2} \mu_{0} \tanh \mu_{0} x
$$

with $\mu_{0}$ given by (3.15). It is skew-symmetric with respect to $x=0$ and corresponds via (3.1) and (1.11) to the symmetric density profile

$$
u_{\mathrm{eq}}(x)=2 K^{2} \mu_{0}^{2} / \cosh ^{2} \mu_{0} x
$$


the latter being the unique equilibrium solution of the original parabolic problem (1.24), (1.9).

To analyze the full time dependent problem (3.2)-(3.5) it is convenient to employ the Hopf-Cole transformation; that is, to introduce a positive potential $\varphi$ via the relations:

$$
\frac{\partial \varphi}{\partial x}=\frac{W}{2 K^{2}} \varphi \quad \text { and } \quad \frac{\partial \varphi}{\partial t}=\frac{-\left[B(t) W-W^{2} / 2-K^{2}(\partial W / \partial x)\right]}{2 K^{2}} \varphi .
$$

It is easily checked that if $W$ satisfies (3.2), then the relations (3.18) are compatible; that is that $\partial^{2} \varphi / \partial x \partial t=\partial^{2} \varphi / \partial t \partial x$. It is also easily checked that

$$
W=2 K^{2} \frac{\partial \log \varphi}{\partial x}
$$

satisfies (3.2)-(3.5), if $\varphi$ evolves as

$$
\frac{\partial \varphi}{\partial t}-K^{2} \frac{\partial^{2} \varphi}{\partial x^{2}}=-B(t) \frac{\partial \varphi}{\partial x}
$$

where $B$ is given by

$$
B(t)=\frac{K^{2} \Lambda}{1+\Lambda} \log \left(\frac{\varphi(1, t)}{\varphi(-1, t)}\right)
$$

and $\varphi$ satisfies

$$
\frac{\partial \varphi}{\partial x}(-1, t)=\frac{-\bar{u}}{2 K^{2}} \varphi(-1, t) \quad \text { and } \quad \frac{\partial \varphi}{\partial x}=\frac{\bar{u}}{2 K^{2}} \varphi(1, t),
$$

as well as the initial condition

$$
\varphi\left(x, 0^{+}\right)=\exp \left(\frac{1}{2 K^{2}} \int_{-1}^{x} \hat{W}(\xi) d \xi\right) \stackrel{\text { def }}{=} \hat{\varphi}(x)>0, \quad-1<x<1 .
$$

The important thing to note about the initial data for $\varphi$ is that it is compatible with the boundary conditions (3.22). This follows from constraints imposed on $\hat{U}$ in (1.29).

To get a better understanding of the structure of the problem (3.20)-(3.23) it is convenient to write $\varphi$ as

$$
\varphi(x, t)=\varphi_{1}(x, t)+\varphi_{2}(x, t)
$$

where $\varphi_{1}$ is odd and $\varphi_{2}$ is even in $x$. A straightforward calculation then shows that $\varphi$ solves (3.7)-(3.10), if and only if $\varphi_{1}$ and $\varphi_{2}$ satisfy

$$
\begin{aligned}
& \left\{\begin{array}{l}
\frac{\partial \varphi_{1}}{\partial t}-K^{2} \frac{\partial^{2} \varphi_{1}}{\partial x^{2}}=-B(t) \frac{\partial \varphi_{2}}{\partial x}, \\
\frac{\partial \varphi_{2}}{\partial t}-K^{2} \frac{\partial^{2} \varphi_{2}}{\partial x^{2}}=-B(t) \frac{\partial \varphi_{1}}{\partial x},
\end{array}\right. \\
& B(t)=\frac{K^{2} \Lambda}{1+\Lambda} \log \left(\frac{\varphi_{2}(1, t)+\varphi_{1}(1, t)}{\varphi_{2}(1, t)-\varphi_{1}(1, t)}\right), \\
& \left\{\begin{array}{l}
\varphi_{1}(0, t)=0 \quad \text { and } \quad \frac{\partial \varphi_{1}}{\partial x}(1, t)=\frac{\bar{u}}{2 K^{2}} \varphi_{1}(1, t), \\
\frac{\partial \varphi_{2}}{\partial x}(0, t)=0 \text { and } \quad \frac{\partial \varphi_{2}}{\partial x}(1, t)=\frac{\bar{u}}{2 K^{2}} \varphi_{2}(1, t),
\end{array}\right.
\end{aligned}
$$




$$
\begin{aligned}
& \varphi_{1}\left(x, 0^{+}\right)=\frac{1}{2}(\hat{\varphi}(x)-\hat{\varphi}(-x)) \\
& \varphi_{2}\left(x, 0^{+}\right)=\frac{1}{2}(\hat{\varphi}(x)+\hat{\varphi}(-x)), \quad 0<x<1 .
\end{aligned}
$$

Our first result is

THEOREM 1. The manifold $\varphi_{1} \equiv 0$ is invariant. On this manifold $B(t) \equiv 0$ and $\varphi_{2}$ satisfies

$$
\begin{aligned}
& \frac{\partial \varphi 2}{\partial t}-K^{2} \frac{\partial^{2} \varphi_{2}}{\partial x^{2}}=0, \quad 0<x<1 \quad \text { and } t>0, \\
& \frac{\partial \varphi_{2}}{\partial x}(0, t)=0 \quad \text { and } \quad \frac{\partial \varphi_{2}}{\partial x}(1, t)=\frac{\bar{u}}{2 K^{2}} \varphi_{2}(1, t),
\end{aligned}
$$

and

$$
\varphi_{2}\left(x, 0^{+}\right)=\frac{1}{2}(\hat{\varphi}(x)+\hat{\varphi}(-x)), \quad 0<x<1 .
$$

The solution on this manifold is given by

$$
\varphi_{2}=\beta_{0} e^{\mu_{0}^{2} K^{2} t} \Psi_{0}(x)+\sum_{n=1}^{\infty} \beta_{2 n} e^{-\mu_{2 n}^{2} K^{2} t} \Psi_{2 n}(x)
$$

where $\mu_{0}>0$ is as in (3.15) and

$$
\left(\frac{2 n-1}{2}\right) \pi<\mu_{2 n}<n \pi \text { is such that } \cot \mu_{2 n}=\frac{-2 \mu_{2 n} K^{2}}{\bar{u}}
$$

$$
\Psi_{0}(x)=A_{0} \cosh \mu_{0} x \text { and } A_{0}>0 \text { is such that }
$$

$$
\int_{0}^{1} \Psi_{0}^{2}(x) d x=1
$$

$$
\Psi_{2 n}(x)=A_{2 n} \cos \mu_{2 n} x \text { and } A_{2 n}>0 \text { is such that }
$$

$$
\int_{0}^{1} \Psi_{2 n}^{2}(x) d x=1,^{2}
$$

Since $\varphi_{2}\left(x, 0^{+}\right)>0,0<x<1$, we have $\beta_{0}>0$ and hence

$$
W(x, t) \stackrel{\text { def }}{=} \frac{2 K^{2}}{\varphi_{2}(x, t)} \frac{\partial \varphi_{2}}{\partial x}(x, t)
$$

satisfies

$$
\lim _{t \rightarrow \infty} W(x, t)=2 K^{2} \mu_{0} \tanh \mu_{0} x=W_{\mathrm{eq}}(x), \quad 0<x<1,
$$

the unique equilibrium solution; see Proposition 1. Finally, the identities

$$
\left\{\begin{array}{l}
\mu_{0} \sim \bar{u} / 2 K^{2} \quad \text { as } K \rightarrow 0^{+} \\
\mu_{0}^{2} \sim \bar{u} / 2 K^{2} \quad \text { as } K \rightarrow \infty
\end{array}\right.
$$

\footnotetext{
${ }^{2}$ With this normalization the constants $A_{2 n}$ are $O(1)$ and $\lim _{n \rightarrow \infty} A_{2 n}=\sqrt{2}$.
} 
imply that

$$
\lim _{K \rightarrow 0^{+}} W_{\mathrm{eq}}(x)=\bar{u}, \quad 0<x<1
$$

and

$$
\lim _{K \rightarrow \infty} W_{\text {eq }}(x)=\bar{u} x, \quad 0<x<1 .
$$

PROOF. The assertions (3.29)-(3.31) follow directly from (3.25)-(3.27) while the representation formula (3.32) together with (3.33)-(3.36) is an elementary consequence of separating variables. The positivity of the data $\varphi_{2}\left(x, 0^{+}\right)$follows from (3.23) and this fact, together with the maximum principle, guarantees that $\varphi_{2}(x, t)$ is positive on $0 \leq x \leq 1$ and $t \geq 0$. Thus we are guaranteed that

$$
W(x, t)=\frac{2 K^{2}}{\varphi_{2}} \frac{\partial \varphi_{2}}{\partial x}
$$

is globally defined on the strip $0 \leq x \leq 1$ and $t \geq 0$. That the asymptotic limit (3.37) obtains now follows from the fact that $\beta_{0}=\int_{0}^{1} \varphi_{2}\left(x, 0^{+}\right) \Psi_{0}(x) d x>0$ and from the representation formula (3.32). The relations (3.38) follow directly from (3.15), while (3.39) and (3.40) follow from (3.37) and (3.38).

The remainder of this section will be devoted to the full problem (3.20)-(3.23) when $\varphi_{1}(x, t) \neq 0$. We will reformulate the problem as a system of Volterra integral equations in order to derive a local convergence result (Proposition 2) which will prove the local stability of the equilibrium $W_{\text {eq }}$ (Theorem 2). For this aim we renormalize $\varphi$ and notice that

$$
\phi(x, t)=e^{-\mu_{0}^{2} K^{2}} \varphi(x, t)
$$

has to fulfill the equations

$$
\begin{aligned}
& \frac{\partial \phi}{\partial t}-K^{2} \frac{\partial^{2} \phi}{\partial x^{2}}+\mu_{0}^{2} K^{2} \phi=f \\
& \frac{\partial \phi}{\partial x}( \pm 1, t)= \pm \frac{\bar{u}}{2 K^{2}} \phi( \pm 1, t)
\end{aligned}
$$

with

$$
\begin{gathered}
f(x, t)=-B(t) \frac{\partial \phi}{\partial x}(x, t), \\
B(t)=\frac{K^{2} \Lambda}{1+\Lambda} \log \left(\frac{\phi(1, t)}{\phi(-1, t)}\right),
\end{gathered}
$$

and initial value

$$
\phi\left(x, 0^{+}\right)=\hat{\varphi}(x)
$$

For a given source term $f=f(x, t)$ the linear parabolic problem (3.42), (3.43), (3.46) can be solved via explicit construction of Green's Function: 
LEMMA 1. Let

$$
G(x, \xi, t)=\frac{1}{2} \Psi_{0}(x) \Psi_{0}(\xi)+\sum_{m=1}^{\infty} e^{-\lambda_{m} t} \frac{1}{2} \Psi_{m}(x) \Psi_{m}(\xi)
$$

where for $m \geq 2$ :

$$
\lambda_{m}=K^{2}\left(\mu_{0}^{2}+\mu_{m}^{2}\right)
$$

with $\mu_{2 n}$ defined in (3.33) and, analogously,

$$
\begin{aligned}
& (n-1) \pi<\mu_{2 n-1}<\frac{(2 n-1)}{2} \pi \text { is such that } \\
& \mu_{2 n-1} \cot \mu_{2 n-1}=\frac{\bar{u}}{2 K^{2}}, \quad n=2,3, \ldots,
\end{aligned}
$$

with corresponding eigenfunctions $\Psi_{2 n}$ as in (3.34)-(3.35) and, analogously,

$$
\begin{aligned}
& \Psi_{2 n-1}(x)=A_{2 n-1} \sin \mu_{2 n-1} x \text { and } A_{2 n-1}>0 \\
& \text { is such that } \int_{0}^{1} \Psi_{2 n-1}^{2}(x) d x=1 .
\end{aligned}
$$

Finally, for $m=1$

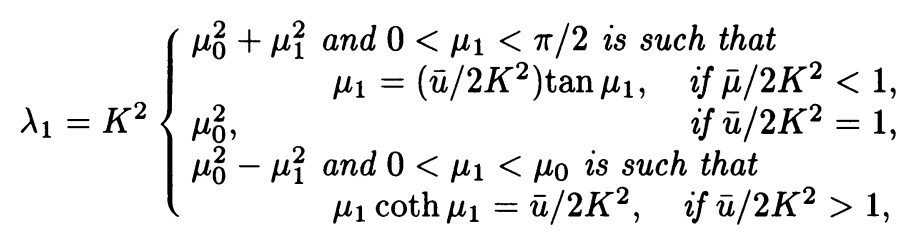

with eigenfunction

$$
\Psi_{1}(x) \equiv\left\{\begin{array}{cc}
A_{1} \sin \mu_{1} x \text { and } A_{1}>0 & \text { is such that } \\
\sqrt{3} x, & \text { if } \bar{u} / 2 K^{2}>1, \\
A_{1} \sin h \mu_{1} x \text { and } A_{1}>0 & \text { is } \begin{array}{l}
\text { such that } \\
\int_{1}^{2}(x) d x=1,
\end{array} \\
\int_{0}^{1} \Psi_{1}^{2}(x) d x=1, & \text { if } \bar{u} / 2 K^{2}>1 .
\end{array}\right.
$$

Then the unique solution of system (3.42), (3.43), (3.46) is given by

$$
\begin{aligned}
\phi(x, t)= & \int_{-1}^{1} G(x, \xi, t) \hat{\varphi}(\xi) d \xi \\
& +\int_{0}^{t} \int_{-1}^{1} G(x, \xi, t-s) f(\xi, s) d \xi d x .
\end{aligned}
$$

Since $\lambda_{m}>0$ for $m=1,2, \ldots$ the Green's function converges towards the projection kernel of the lowest eigenfunction $\Psi_{0}$ with eigenvalue zero:

$$
G(x, \xi, t) \stackrel{t \rightarrow \infty}{\rightarrow} \frac{1}{2} \Psi_{0}(x) \Psi_{0}(\xi) .
$$

This suggests the decomposition

$$
G(x, \xi, t)=\frac{1}{2} \Psi_{0}(x) \Psi_{0}(\xi)+\tilde{G}(x, \xi, t)
$$

and

$$
\phi(x, t)=z_{0}(t) \Psi_{0}(x)+Z(x, t)
$$


with the orthogonality conditions

$$
\int_{-1}^{1} Z(\xi, t) \Psi_{0}(\xi) d \xi=\int_{-1}^{1} \tilde{G}(x, \xi, t) \Psi_{0}(\xi) d \xi=0 .
$$

With the aid of Lemma 1 the nonlinear system (3.42)-(3.46) can then be rewritten as a system of Volterra integral equations;

$$
z_{0}(t)=\hat{\varphi}_{0}-T_{0}\left[B \frac{\partial Z}{\partial x}\right](t)
$$

and

$$
Z(x, t)=\hat{\phi}(x, t)-T_{1}\left[B\left(z_{0} \Psi_{0}^{\prime}+\frac{\partial Z}{\partial x}\right)\right](t, x)
$$

where

$$
\begin{aligned}
B(t) & =\frac{K^{2} \Lambda}{1+\Lambda}\left\{\log \left(1+\frac{Z(1, t)}{\Psi_{0}(1) z_{0}(t)}\right)-\log \left(1+\frac{Z(-1, t)}{\Psi_{0}(1) z_{0}(t)}\right)\right\} \\
& =\frac{K^{2} \Lambda}{1+\Lambda}\left(\frac{Z(1, t)-Z(-1, t)}{\Psi_{0}(1) z_{0}(t)}\right)+\tilde{B}\left(\frac{Z(\cdot, t)}{z_{0}(t)}\right)
\end{aligned}
$$

with

$$
\tilde{B}(\varsigma(\cdot, t))=O\left(\max \varsigma^{2}( \pm 1, t)\right)
$$

and the two linear Volterra operators $T_{i}$ are given by

$$
T_{0}[f](t)=\frac{1}{2} \int_{0}^{t} \int_{-1}^{1} \Psi_{0}(\xi) f(\xi, s) d \xi d s
$$

and

$$
T_{1}[f](t, x)=\int_{0}^{t} \int_{1}^{1} \tilde{G}(x, \xi, t-s) f(\xi, s) d \xi d s .
$$

Furthermore the contributions due to the initial data are

$$
\hat{\varphi}_{0}=\frac{1}{2} \int_{-1}^{1} \Psi_{0}(\xi) \hat{\varphi}(\xi) d \xi
$$

and

$$
\hat{\phi}(x, t)=\int_{-1}^{1} \tilde{G}(x, \xi, t) \hat{\varphi}(\xi) d \xi .
$$

Since an analytic treatment of the full nonlinear problem is beyond us, we shall content ourselves with showing only the following local convergence result.

Proposition 2 (CONVERgence of SMALl Perturbations). Consider system (3.42)-(3.46) for arbitrary initial data near the equilibrium state, i.e.

$$
\hat{\varphi}(x)=\Psi_{0}(x)+O(\alpha), \alpha>0 \text { small. }
$$

Then the unique solution $\phi(x, t)$ converges for $t \rightarrow \infty$ to a multiple of $\Psi_{0}(x)$, more precisely in terms of the decomposition (3.55), there exists a real number $z_{\infty}$ such that

$$
z_{0}(t) \rightarrow z_{\infty}, \quad Z(\cdot, t) \rightarrow 0 \quad \text { as } t \rightarrow \infty
$$


Transforming back to the original functions

$$
W=2 K^{2} \frac{\partial \log \varphi}{\partial x}=2 K^{2} \frac{\partial \log \phi}{\partial x}
$$

and

$$
u=\frac{\partial W}{\partial w}
$$

we deduce from the convergence result in Proposition 2 the following theorem.

THEOREM 2 (LOCAL STABILITY RESULT). The uniquely determined equilibrium $W_{\mathrm{eq}}(3.16)$ of system (3.2)-(3.5) and the corresponding equilibrium solution $u_{\mathrm{eq}}(3.17)$ of the original parabolic problem (1.24) and (1.9) are both locally asymptotically stable, provided the mean value

$$
\bar{u}=\frac{1}{2} \int_{-1}^{1} u_{\mathrm{eq}}(x) d x
$$

is held fixed.

Proof of Proposition 2. Define, as one measure of the "oddness" of $\phi(t, \cdot)$

$$
\beta(t)=Z(1, t)-Z(-1, t)
$$

Then the approximate description of the functional $B$ in (3.59) and (3.58) give us a convolution equation for $\beta$;

$$
\beta(t)+\int_{0}^{t} k(t-s) \beta(s) d s=\gamma(t)
$$

Here the kernel is given by

$$
k(t)=\frac{K^{2} \Lambda}{1+\Lambda} \sum_{n=1}^{\infty} e^{-\lambda_{2 n-1} t} \frac{\Psi_{2 n-1}(1)}{\Psi_{0}(1)} \int_{-1}^{1} \Psi_{2 n-1}(\xi) \Psi_{0}^{\prime}(\xi) d \xi
$$

or

$$
k(t)=\frac{2 K^{4} \Lambda}{1+\Lambda}\left(\mu_{0}^{2}-\left(\frac{\bar{u}}{2 K^{2}}\right)^{2}\right) \sum_{n=1}^{\infty} \frac{\Psi_{2 n-1}(1)^{2}}{\lambda_{2 n-1}} e^{-\lambda_{2 n-1} t}
$$

(this computation directly follows from the properties of the eigenfunctions $\Psi_{0}$ and $\Psi_{2 n-1}$ in (3.15), (3.34) and (3.49)-(3.52)). The right-hand side $\gamma(t)$ in (3.70) depends on $\hat{\phi}, z_{0}$, and $Z$ in the following manner;

$$
\begin{aligned}
\gamma(t)= & \hat{\phi}(1, t)-\hat{\phi}(-1, t)-\int_{0}^{t} k(t-s) \frac{1}{z_{0}(s)} \tilde{B}\left(\frac{Z(\cdot, s)}{z_{0}(s)}\right) d s \\
& -\left[T_{1}\left[B \frac{\partial Z}{\partial x}\right](t, x)\right]_{x=-1}^{x=1} .
\end{aligned}
$$

LEMMA 2. For any given function

$$
\gamma(t)=\gamma_{\infty}+\tilde{\gamma}(t), \quad t \geq 0
$$

with

$$
\sup _{t \geq 0} e^{\rho t}|\tilde{\gamma}(t)| \stackrel{\text { def }}{=}\|\tilde{\gamma}\|_{\rho}<\infty
$$


the convolution equation (3.70) has a unique solution

$$
\hat{B}[\gamma](t) \stackrel{\text { def }}{=} \beta(t)=\gamma(t)-\int_{0}^{t} r(t-s) \gamma(s) d s, \quad t \geq 0,
$$

which satisfies a uniform estimate

$$
\|\beta\|_{\rho} \leq G_{1}\left|\gamma_{\infty}\right|+G_{2}\|\tilde{\gamma}\|_{\rho}, \quad \text { provided } 0<\rho \leq \lambda_{1} .
$$

Here $r(\cdot)$ is the resolvent kernel satisfying

$$
r(t)+\int_{0}^{t} k(t-s) r(s) d s=k(t), \quad t \geq 0 .
$$

PROOF. The Laplace transforms $\hat{k}$ and $\hat{r}$ of $k$ and $r$ are related by

$$
\hat{r}(z)=\hat{k}(z) /(1+\hat{k}(z))
$$

where from (3.71) we compute

$$
\hat{k}(z)=\frac{2 K^{4} \Lambda\left(\mu_{0}^{2}-\left(\bar{u} / 2 K^{2}\right)^{2}\right)}{1+\Lambda} \sum_{n=1}^{\infty} \frac{\Psi_{2 n-1}^{2}(1)}{\lambda_{2 n-1}} \frac{1}{z+\lambda_{2 n-1}} .
$$

$\hat{k}$ has poles at points

$$
z_{n}=-\lambda_{2 n-1} .
$$

From the fact that the residues of $\hat{k}$ at the points $z_{n}$ are positive and thus

$$
\lim _{x(\text { real }) \rightarrow \pm z_{n}} \hat{k}(x)= \pm \infty,
$$

it follows that $\hat{r}$ has poles at points

$$
p_{n}=-r_{n}
$$

where

$$
\lambda_{2 n-1}<r_{n}<\lambda_{2 n+1} \quad n=1,2, \ldots
$$

Then the inversion formula for the Laplace transform yields: For any $0<\rho<r_{1}$ there are constants $0<R_{\rho}<\infty$ such that the following bounds obtain:

$$
\|r\|_{\rho} \leq R_{\rho}
$$

and

$$
\left\|\int^{\infty} r(s) d s\right\|_{\left(\rho+r_{1}\right) / 2} \leq \frac{2 R_{\left(\rho+r_{1}\right) / 2}}{K^{2}\left(\rho+r_{1}\right)} .
$$

Using (3.73) and (3.75) this finally implies the desired estimate (3.76):

$$
\|\beta\|_{\rho} \leq \frac{2 R_{\left(\rho+r_{1}\right) / 2}}{K^{2}\left(\rho+r_{1}\right)}\left|\gamma_{\infty}\right|+\left(1+\frac{2 R_{\left(\rho+r_{1}\right) / 2}}{K^{2}\left(r_{1}-\rho\right)}\right)\|\tilde{\gamma}\|_{\rho}
$$

where the constants are uniformly bounded for $0<\rho \leq \lambda_{1}<r_{1}$.

With the aid of Lemma 2 we now can finish the proof of Proposition 2 by using the Banach fixed point theorem and standard regularity estimates for Green's 
function $\tilde{G}$. We define the following norms for the functions $z_{0}(t), \beta(t)$ and $\zeta(x, t)=$ $\partial Z(x, t) / \partial x$

$$
\begin{gathered}
\left\|z_{0}\right\|_{0}=\sup _{t \geq 0}\left|z_{0}(t)\right| \\
\|\beta\|_{\rho}=\sup _{t \geq 0}\left\{e^{\rho t}|\beta(t)|\right\} \\
\|\|_{\zeta} \|_{\rho}=\sup _{t \geq 0}\left\{e^{\rho t}\|\zeta(\cdot, t)\|_{L^{2}[-1,1]}\right\},
\end{gathered}
$$

and remark that condition (3.56) and $\Psi_{0}>0$ imply

$$
\|Z( \pm 1, \cdot)\|_{\rho} \leq C \mid\left\|\frac{\partial Z}{\partial x}\right\| \|_{\rho} .
$$

From the initial condition (3.65) and the definition of $\tilde{G}$ in (3.47) and (3.54) we conclude for $\hat{\varphi}_{0}$ in (3.63) and for $\hat{\phi}$ in (3.64) the estimates

$$
\left|\hat{\varphi}_{0}-1\right|=O(\alpha)
$$

and

$$
\|\hat{\phi}\|_{\rho}=O(\alpha) \text { for } \rho \leq \lambda_{1} .
$$

Moreover the operator $T_{0}$, see (3.61), has the obvious property

$$
\left\|T_{0}[f]\right\|_{\rho} \leq C_{\rho}\|f \mid\|_{\rho} \text { for all } \rho>0
$$

and careful estimates yield an analogous result for $T_{1}$, see (3.62),

$$
\left\|T_{1}[f]( \pm 1, \cdot)\right\|_{\rho} \leq C_{\lambda_{1}-\rho}\|f \mid\|_{\rho}
$$

and even for its first derivative (see Lemma 3):

$$
\left\|\frac{\partial}{\partial x} T_{1}[f]\right\|\left\|_{\rho} \leq C_{\lambda_{1}-\rho}\right\| f \mid \|_{\lambda_{1}}
$$

but only for $\rho<\lambda_{1}$. Here we have used the fact that the eigenfunctions $\Psi_{m}$ fulfill uniform estimates

$$
\left|\Psi_{m}( \pm 1)\right| \leq C, \quad m \geq 0
$$

and

$$
\lambda_{m}^{-1}\left\|\Psi_{m}^{\prime}\right\|_{L^{2}}^{2} \leq C, \quad m \geq 1 .
$$

Consider now the augmented Volterra integral system (3.57), (3.58) and (3.70) for $z_{0}, Z$, and $\beta$ with $B$ (see (3.59)), being linear in $\beta$ and quadratic in $Z( \pm 1, \cdot)$ and $\gamma$ (see (3.72)), and being quadratic in $(\beta, Z( \pm 1, \cdot), \partial Z / \partial x)$. According to the inequalities (3.85), (3.89)-(3.94) we obtain the following a priori estimates for any $\rho$ satisfying

$$
\frac{\lambda_{1}}{2} \leq \rho<\lambda_{1}
$$


namely

$$
\begin{aligned}
& \left\|z_{0}-1\right\|_{0}=O\left\{\alpha+\left(\|\beta\|_{\rho}+\left\|\frac{\partial Z}{\partial x}\right\|_{\rho}\right)\left\|\frac{\partial Z}{\partial x}\right\| \|_{\rho}\right\} \\
& \left.\left\|\frac{\partial Z}{\partial x}\right\|\right|_{\rho}=O\left\{\alpha+\|\beta\|_{\rho}+\left\|\frac{\partial Z}{\partial x}\right\| \|_{\rho}^{2}\right\}
\end{aligned}
$$

and

$$
\|\beta\|_{\rho}=O\left\{\alpha+\left(\|\beta\|_{\rho}+\left\|\frac{\partial Z}{\partial x}\right\| \|_{\rho}\right)\left\|\frac{\partial Z}{\partial x}\right\| \|_{\rho}\right\}
$$

The usual application of Banach's fixed point theorem then yields the unique existence of a solution of system (3.57), (3.58) satisfying the estimates

$$
\left\|\frac{\partial Z}{\partial x}(\cdot, t)\right\|_{L^{2}}=e^{-\rho t} O(\alpha)
$$

and

$$
\left|z_{0}(t)\right|=1+O(\alpha)
$$

Moreover, (3.98) and (3.57) even imply the existence of the limit

$$
z_{\infty} \stackrel{\text { def }}{=} \lim _{t \rightarrow \infty} z_{0}(t)=\hat{\varphi}_{0}+O(\alpha)=1+O(\alpha)
$$

so that the convergence statement (3.66) in Proposition 2 follows, where convergence of $Z(\cdot, t)$ takes place in the Sobolev space $H^{1,2}[-1,1]$. In general $H^{m, 2}$ denotes the space of functions whose $m$ th derivatives are $L^{2}$-integrable.

REMARK. Regarding the compatibility condition (1.22) at the boundary one can improve the regularity estimates and prove at least the $H^{2,2}$-convergence of $Z(\cdot, t)$ in Proposition 2, which then implies the local $H^{1,2}$-stability of $W_{\text {eq }}$ and the $L^{2}$-stability of $u_{\text {eq }}$ in Theorem 2 .

The proof of Proposition 2 depends on the basic estimates (3.92)-(3.94), where the last one is the most difficult and whose derivation we would like to sketch briefly. We state

LEMMA 3. Let $f(\xi, t)=\sum_{m=0}^{\infty} c_{m}(t) \Psi_{m}(\xi)$ be the square summable Fourier representation of a time dependent $L^{2}$-function $f(\cdot, t)$. Then for each $0<\rho<\lambda_{1}$ there is a uniform positive constant $C_{\lambda_{1}-\rho}$ such that

$$
\begin{aligned}
& e^{2 \rho t} \int_{-1}^{1}\left(\int_{0}^{t} \int_{-1}^{1} \frac{\partial}{\partial x} G(x, \xi, t-s) f(\xi, s) d \xi d s\right)^{2} d x \\
& \quad \leq C_{\lambda_{1}-\rho} \int_{0}^{t} \sum_{m=1}^{\infty} c_{m}^{2}(s) e^{2 \rho s} d s .
\end{aligned}
$$

The proof uses the Cauchy-Schwarz-inequality in order to derive an estimate of the left-hand side by

$$
\sup _{m \geq 1}\left|\int_{0}^{t} \lambda_{m} e^{-2 \lambda_{m}(t-s)} e^{2 \rho(t-s)} d s\right| \int_{0}^{t} \sum_{m=1}^{\infty} \lambda_{m}^{-1}\left(\int_{-1}^{1}\left|\Psi_{m}^{\prime}(x)\right|^{2} d x\right) c_{m}^{2}(s) e^{2 \rho s} d s
$$

which gives the desired bound for each $\rho<\lambda_{1} \leq \lambda_{m}$, regarding that the $H^{1,2}$-norm of the eigenfunctions $\Psi_{m}$ is $O\left(\sqrt{\lambda_{m}}\right)$, see (3.96). 

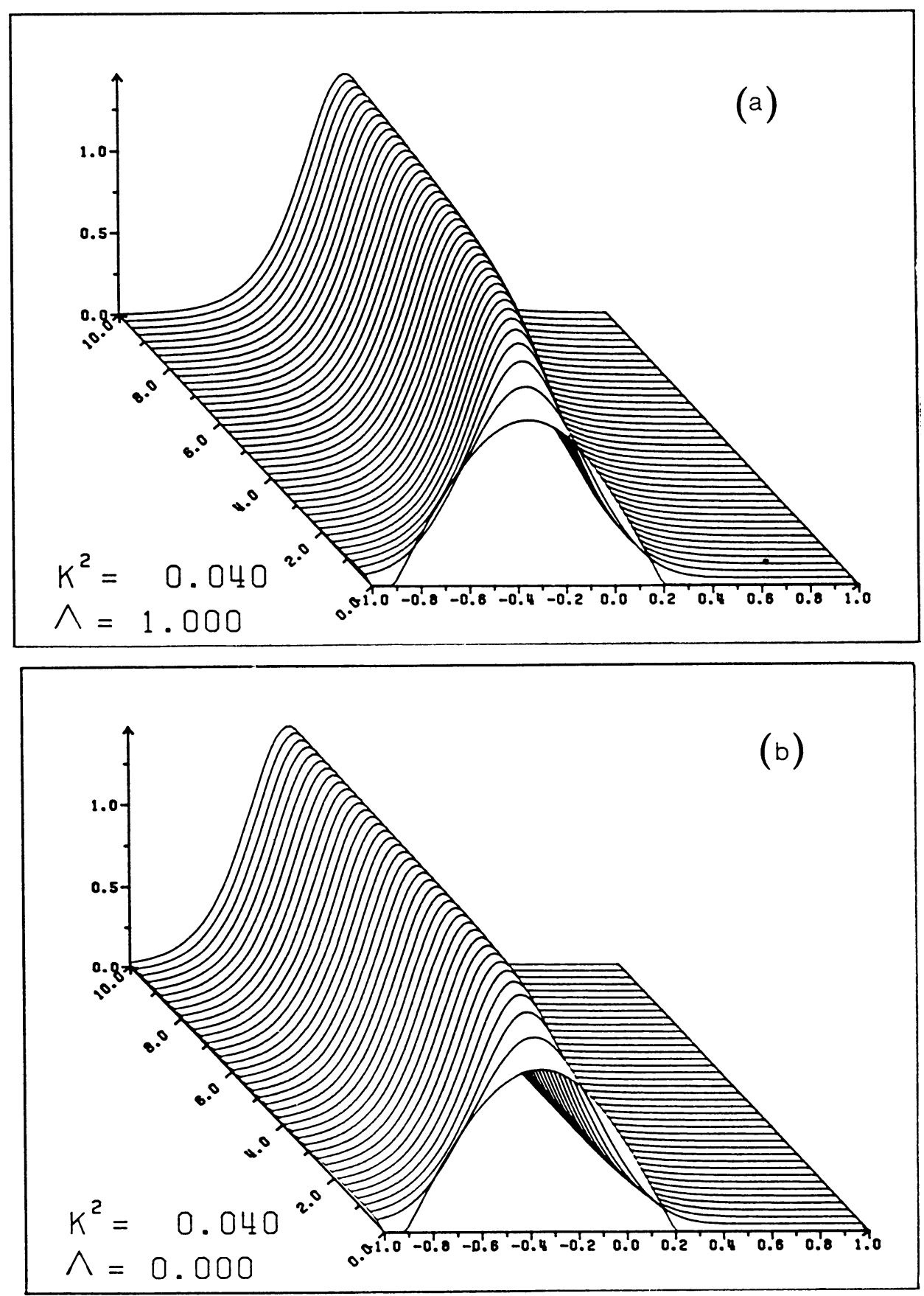

FIGURE 2. Numerical simulations of the time evolution $u=u(t, x)$, $0 \leq t \leq 10$, for $K^{2}=0.04$ and different $\Lambda$. (a) For $\Lambda=1$ a stable aggregation profile is formed, identical to $u_{\text {eq }}$. (b) For $\Lambda=0$ a "pseudo steady state" of similar shape develops, whose position depends on the initial distribution. (It slowly moves to the origin as in (a), but for much larger times $t>200$.) 


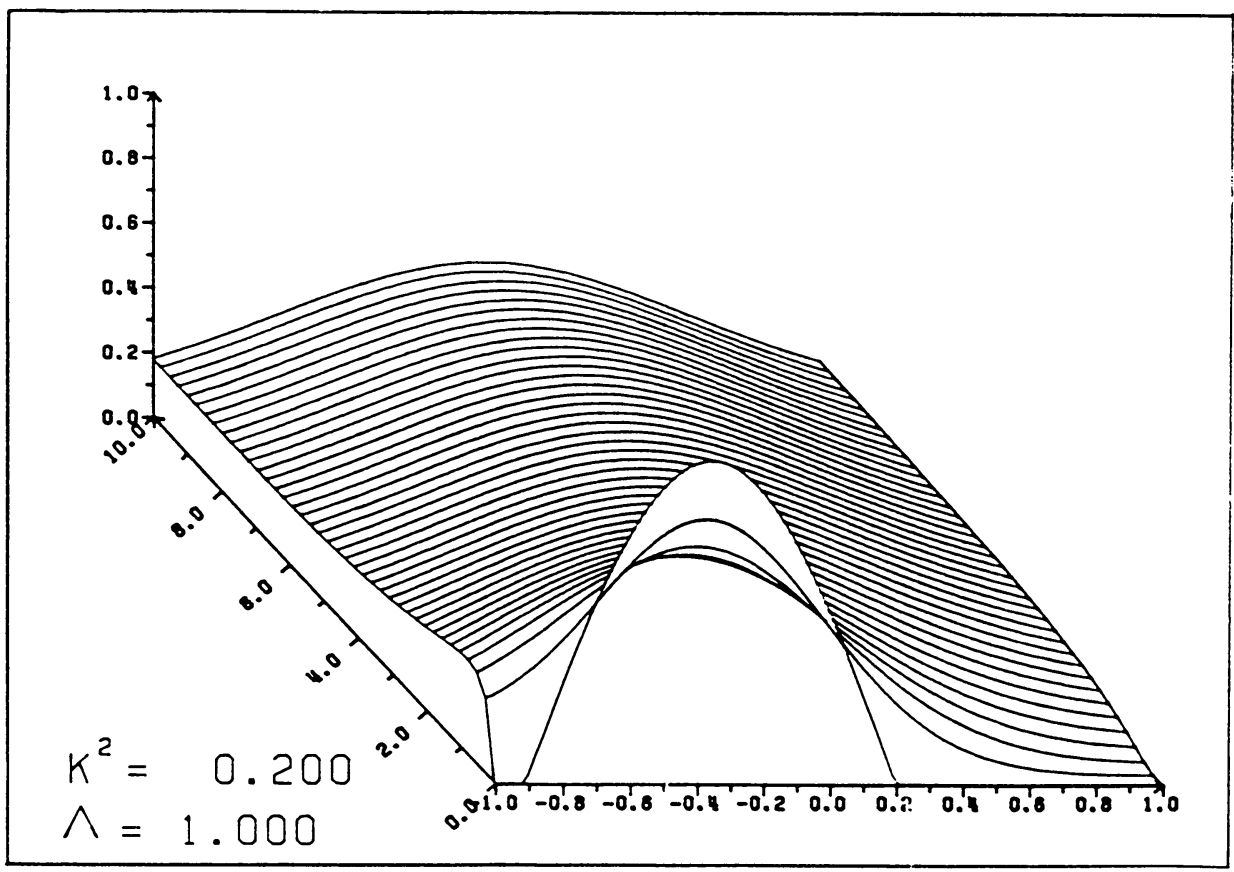

FIGURE 3. The same simulation as in Figure 2a; but with larger diffusion constant $K^{2}=0.2$, showing a broad final aggregation profile.

From Lemma 3 we conclude

$$
\left\|\frac{\partial}{\partial x} T_{1}[f]\right\|\left\|_{\rho} \leq C_{\lambda_{1}-\rho, \hat{\rho}-\rho}\right\| f f \|_{\hat{\rho}}
$$

for all $\rho<\min \left(\lambda_{1}, \hat{\rho}\right)$. Taking $\hat{\rho}=\lambda_{1}$ we finally arrive at the estimate (3.94).

4. Numerical investigations. We use a semidiscretization of the spatial domain $[-1,1]$ into $N$ equal intervals $\left.I_{i}=\right] x_{i-1}, x_{i}$ [ with piecewise constant approximations for the density

$$
u(t, x) \sim u_{i}(t) \quad \text { for all } x \in I_{i}
$$

and piecewise linear approximations for the cumulative density function $W=U-\bar{u}$ in $(3.1)$ in such a way that

$$
W\left(t, x_{i}\right) \sim W_{i}(t)
$$

with the discretized property $\partial W / \partial x=u$, namely

$$
(N / 2)\left(W_{i}-W_{i-1}\right)=u_{i} \text { for } i=1, \ldots, N .
$$

According to (3.4) we get the side conditions

$$
W_{0}=-\bar{u}, \quad W_{N}=\bar{u},
$$

where the discrete mean value in analogy to (1.14) has to be taken as

$$
\vec{u}=\frac{1}{N} \sum_{i=1}^{N} u_{i}
$$



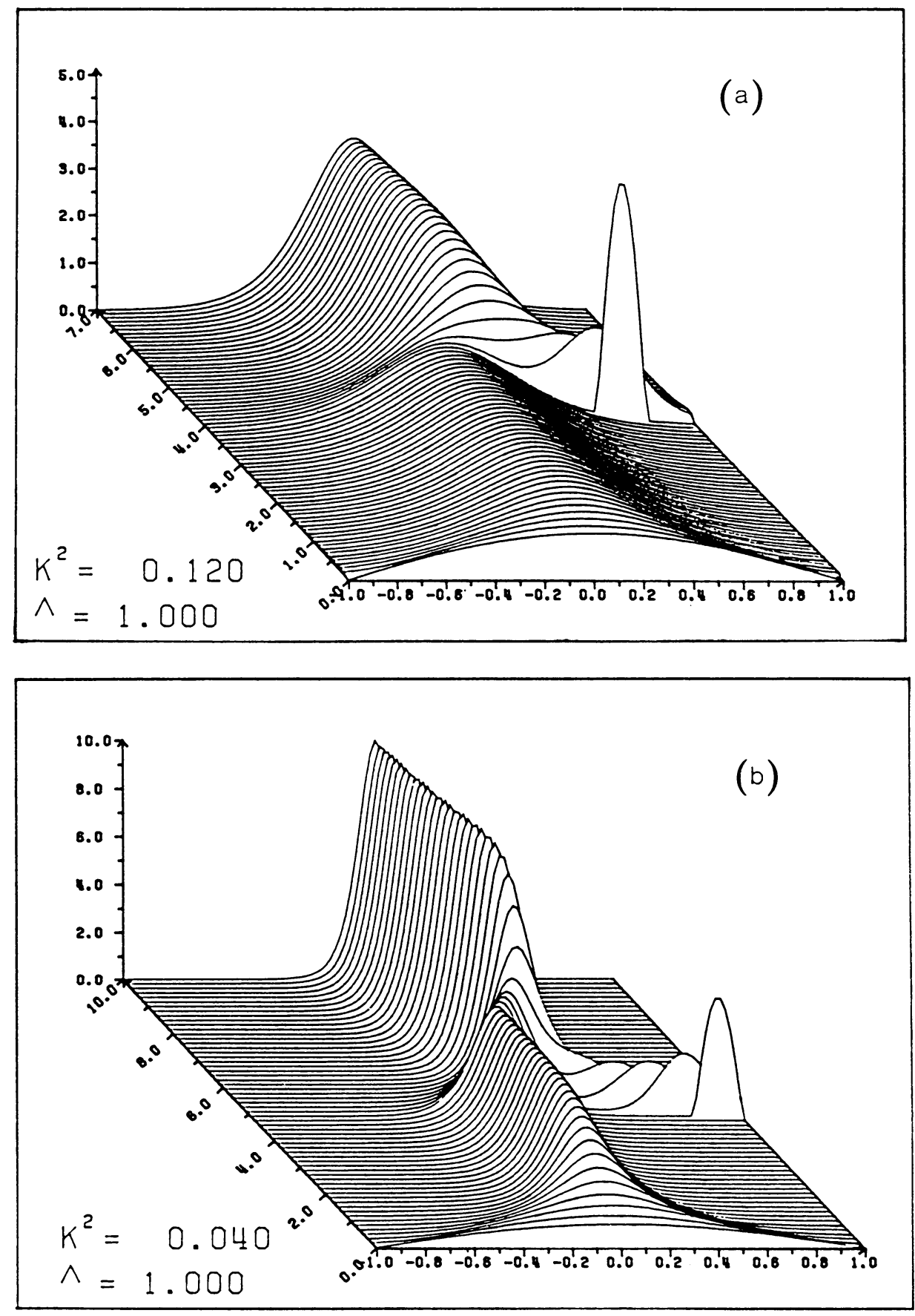

FIGURE 4. Stable aggregation profiles $u_{\text {eq }}$ developing from a broad initial distribution, are perturbed by a secondary hump, for larger (a) and smaller (b) diffusion constant $K^{2}, \Lambda=1$. After the perturbation the two humps coalesce to a new aggregation profile $u_{\text {eq }}^{1}$, which is larger and more centered around the origin. 

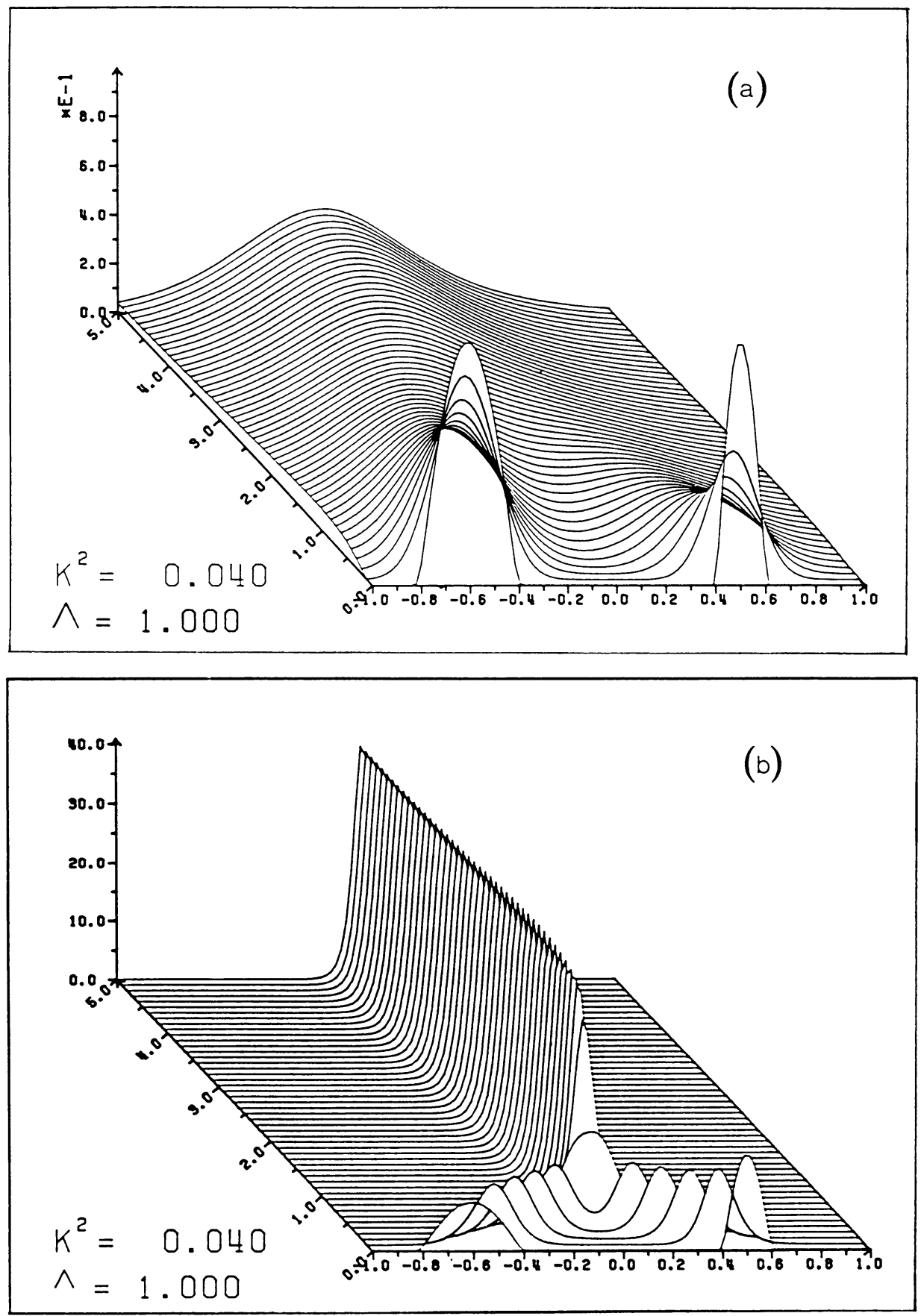

FIGURE 5. The merging process of two, initially separated humps: (a) With amplitude $\sim 1$ and $\bar{u} \sim 0.2$, leading to initial dispersion and slow aggregation into a broad final distribution. (b) With amplitude $\sim 10$ and $\bar{u} \sim 2$, showing initial formation of two separated "centers" which rapidly attract each other and finally form a sharp aggregation profile. 
Then the standard upwind difference scheme for the parabolic equation (3.2) consists in the following ODE-system for $W=\left(W_{i}\right), i=1, \ldots, N-1$;

$$
\dot{W}_{i}=-V_{i} D_{i} W+\frac{1}{4} N^{2} K^{2}\left(W_{i+1}-2 W_{i}+W_{i-1}\right)
$$

with "characteristic velocities"

$$
V_{i}(t)=B(t)-W_{i}(t)
$$

and the upwind difference operator

$$
D_{i} W(t)= \begin{cases}u_{i+1}(t) & \text { if } V_{i}(t)<0 \\ u_{i}(t) & \text { if } V_{i}(t) \geq 0\end{cases}
$$

Here the $u_{i}$ are given by (4.3) and the functional $B(t)$ approximating $B(t)$ in (3.3) simply is

$$
B(t)=\frac{\Lambda}{1+\Lambda} \frac{1}{N} \sum_{i=1}^{N-1} W_{i}(t)
$$

since the side terms in (4.4) cancel each other.

An asymptotic equilibrium is attained for large $t$ in all numerical simulations with any chosen initial data. For $\Lambda>0$ the "final" shape of the equilibrium distribution fits the theoretical values: compare Figure $2 \mathrm{a}$ with Figure $1 \mathrm{~b}$ and Figure 3 with Figure 1a.

We also performed the corresponding numerical simulations in the case $\Lambda=0$. Within the same time a "pseudo steady state" develops which is not centralized, see Figure $2 \mathrm{~b}$. However, for $t \rightarrow \infty$ this hump slowly moves to the origin as predicted.

More generally, for $\Lambda \geq 0$, we observe that the convergence to the unique equilibrium $u_{\text {eq }}$ is faster in time the larger we choose the parameter $\Lambda$, i.e. essentially the parameter $\Lambda /(1+\Lambda)$ according to (4.9).

The two simulations in Figure 4 illustrate that even large perturbations $u_{0}=$ $u_{\text {eq }}+u_{1}$ of the equilibrium state converge quite rapidly to the new equilibrium $u_{\text {eq }}^{1}$ which is uniquely determined by the condition for the initial mean value

$$
\overline{u_{\text {eq }}^{1}}=\overline{u_{\text {eq }}}+\overline{u_{1}}, \quad \overline{u_{1}}>0 .
$$

Comparison of the shapes of $u_{\mathrm{eq}}^{1}$ and $u_{\mathrm{eq}}$ confirms the theoretical information from Proposition 1, that the final density distribution is more centered around the origin for larger mean value $\bar{u}$ (see also Figure 1 ).

This can clearly be seen in Figure 5 where two small humps, initially separated, move towards each other in a different manner, until they finally coalesce.

\section{REFERENCES}

1. W. Alt, Models for mutual attraction and aggregation of motile individuals, Proc. Conf. Mathematics in Biology and Medicine (July 1983, Bari), Lecture Notes in Biomath., vol. 57, Springer, 1985, pp. 33-38.

2. __ Degenerate diffusion equations with drift functionals modelling aggregation, Nonlinear Anal. Theory, Methods and Applications 9 (1985), 811-836.

3. W. Alt and D. A. Lauffenburger, Transient behavior of a chemotaxis system modelling certain types of tissue inflammation, Preprint \#305, SFB 123, Heidelberg, 1985. (Submitted to J. Math. Biol.) 
4. S. Childress and J. K. Percus, Nonlinear aspects of chemotaxis, Math. Biosci. 56 (1981), 217-237.

5. G. Gerisch, Cell aggregation and differentiation in dictyostelium, Current Topics in Develop. Biol., vol. 3, 1968, pp. 157-197.

6. E. F. Keller and L. A. Segal, Model for chemotaxis, J. Theoret. Biol. 30 (1971), 225-234.

7. P. Lax, Hyperbolic systems of conservation laws and the mathematical theory of shock waves, C.B.M.S. Regional Conf. Ser. Appl. Math., No. 11, SIAM, Philadelphia, Pa., 1973.

8. T. Nagai and M. Mimura, Some nonlinear degenerate diffusion equations related to population dynamics, J. Math. Soc. Japan 35 (1983), 539-562.

9. __ Asymptotic behavior of the interfaces to a nonlinear degenerate diffusion equation in population dynamics, preprint, 1985.

10. L. A. Segel, Mathematical models for cellular behavior, Studies in Mathematical Biology (S. Levin, ed.), Vol. 15, MAA, Washington, D.C., 1978, pp. 156-190.

11. J. Smoller, Shock waves and reaction-diffusion equations, Springer-Verlag, Berlin, Heidelberg and New York, 1983.

12. R. Schaaf, Stationary solutions of chemotaxis systems, Trans. Amer. Math. Soc. 292 (1986), 531-556.

Department of Mathematics, Ohio State University, Columbus, Ohio 43210

SONDERForschungSBereich 123, UNiversität Heidelberg, D-6900 Heidelberg, Federal Republic of Germany (Current address of Wolfgang Alt)

Current address (J. M. Greenberg): Department of Mathematics, University of Maryland, Baltimore County, Catonsville, Maryland 21228 